\title{
Research Paper \\ The Effect of Gender and Attentional Focus on Postural Control in the Elderly
}

\author{
*Mandana Sangari ${ }^{1} \oplus$, Seyed Mohammadkazem Vaez Mousavi², Parvaneh Shamsipour Dehkordi ${ }^{3}$ Mahdi Namazizadeh ${ }^{4}$ \\ 1. Department of Movement Behavior, Faculty of Physical Education and Sport Science, Central Tehran Branch, Islamic Azad University, Tehran, Iran. \\ 2. Department of Physical Education and Sport Sciences, Imam Hossein University, Tehran, Iran. \\ 3. Department of Movement Behavior, Faculty of Physical Education, Alzahra University, Tehran, Iran \\ 4. Department of Physical Education and Sport Science, Faculty of Physical Education \& Sports Sciences, Khorasgan Branch, Islamic Azad University, Esfahan, Iran.
}

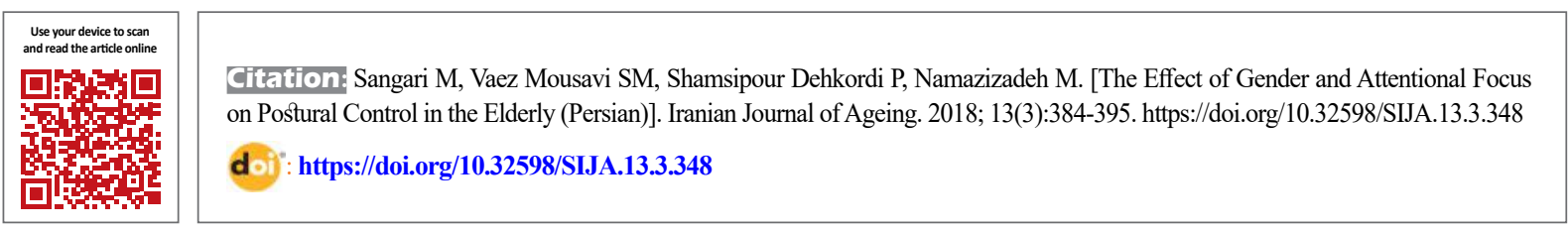

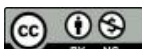

Funding: See Page 392

Received: 16 Mar 2018

Accepted: 21 Jul 2018

Keywords:

Balance, Attention,

Cognitive function,

Gender difference,

Elderly

\section{A B STRACT}

Objectives This study aimed to compare the effects of sex and different types of attentional focus of supra-postural tasks on postural control learning in older adults.

Methods \& Materials A total of 80 older adults (mean [SD] age: 72.5[4.9] year) participated in this study. The participants, selected according to inclusion criteria, were randomly divided into eight groups of internal and external attentional focus based on supra-postural cognitive and motor task guidelines. The postural status was evaluated by measuring the centre of gravity alignment through a master balancing system (modified clinical test of sensory interaction and balance).

Results The results showed that the men's older adults with external focus in motor supra-postural task, had a better posture performance than the other groups $(P<0.05)$. Women older adults with internal focus in cognitive supra-postural tasks had the weakest status in postural control function. Men and women also had a better postural control function than the other test groups in external attentional focus.

Conclusion Postural control loss is greater in older women than men according to the impact of focusing attention on postural control. It is possible to improve the balance by planning external focus in suprapostural task programs.

\section{Extended Abstract}

\section{Objectives}

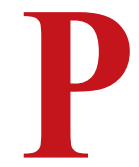

ostural control is essential to maintain stability in static and dynamic activities [1]. Any conscious attention to postural control increases the likelihood of instability [2]. Reinvestment theory suggests that internal attention to motor control can disrupt its processes [3]. Aging is one of the factors that increases the conscious processing [4]. The use of attention instructions in postural and supra-postural tasks can improve postural control [5-7].

Several studies have also been conducted on the comparison of gender effects on the effectiveness of the focus of attention instructions for cognitive and motor tasks. There are no consistent views to provide a general theory. Some observations have reported that

\section{* Corresponding Author:}

Mandana Sangari, PhD Candidate

Address: Department of Movement Behavior, Faculty of Physical Education and Sport Science, Central Tehran Branch, Islamic Azad University, Tehran, Iran.

Tel: +98 (935) 1905634

E-mail: mandana.sangari@gmail.com 
the external focus of attention are more beneficial, and others have observed no difference in the internal and external focus of attention. The present study seeks to investigate whether postural control responses to the focus of attention instructions of cognitive and motor tasks are different among elderly men and women.

\section{Methods and Materials}

The participants were 80 elderly (40 men and 40 women) with a mean (SD) age of 72.50(4.9) years. They were randomly assigned to 8 experimental groups. Sample size was determined based on GPower software. The participants were healthy without orthopedic and neurological disorders. This study has been approved by the Research Ethics Committee of the Department of Physical Education and Sport Sciences at Islamic Azad University, West Tehran Branch, Tehran, Iran. The Sensory Organization Test (SOT) balance master system was used to measure postural status of the participants [8].

Motor task required the participants to hold a wooden tube with both hands at abdomen-height, with the elbows flexed at 90 degrees. A table tennis ball was placed inside of the tube. When contacting the right or left side of the tube, a distinctive sound was heard allowing the experimenter to count the number of contacts. This was considered as a sign of external focus of attention in postural task [9]. Cognitive task with internal focus of attention instructions included counting backward from 100 to 3 [10].
Cognitive task with external focus of attention instructions refers to the identification of a signal that includes vertical lines provided by the software located on the laptop screen at a distance of $1 \mathrm{~m}$ from the participants. One day before the training session, after the explanation about how to do the work, the subjects were divided into two groups of internal and external attentional focuses, and then each group was trained and tested with respect to cognitive and motor tasks.

To analyze the obtained data, descriptive statistics including mean and standard deviation, Shapiro-Wilk test to examine the normality of data, Levene's test to determine the equality of variances, 1-way ANOVA to determine the difference between the data in the pretest phase and the retention test, and Duncan's post hoc test to examine the place of difference in the groups, were used. All statistical tests were performed in SPSS version 20 at a significant level of 0.05 .

\section{Result}

Shapiro-Wilk test results showed that the distribution of data for the Center of Gravity (COG) position was normal $(\mathrm{P}=0.10)$. Levene's test results indicated equal variances for the data of $C O G$ position $(\mathrm{P}=0.38)$. One-way ANOVA results in pretest phase revealed that there was no significant difference between the mean position of the COG in the eight experimental groups where $\mathrm{f}_{(79,7)}=1061$ and $\mathrm{P}=0.147$.

Table 1. Duncan's post hoc test results for paired comparison of the mean position of COG in the eight experimental groups in the retention test

\begin{tabular}{|c|c|c|c|c|c|}
\hline \multirow{2}{*}{ Groups } & \multirow{2}{*}{ No. } & \multicolumn{4}{|c|}{$\alpha=0.05$} \\
\hline & & $1^{\text {st }}$ Column & $2^{\text {nd }}$ Column & $3^{\text {rd }}$ Column & $4^{\text {th }}$ Column \\
\hline Men-external attentional focus-motor task & 10 & 0.28 & & & \\
\hline Men-external attentional focus-cognitive task & 10 & 0.34 & 0.34 & & \\
\hline Women-external attentional focus-motor task & 10 & 0.37 & 0.37 & & \\
\hline Men-internal attentional focus-motor task & 10 & 0.64 & 0.64 & 0.64 & \\
\hline Women-external attentional focus-cognitive task & 10 & 0.73 & 0.73 & 0.73 & 0.73 \\
\hline Men-internal attentional focus-cognitive task & 10 & & 0.78 & 0.78 & 0.78 \\
\hline Women-internal attentional focus-motor task & 10 & & & 0.85 & 0.85 \\
\hline Women-internal attentional focus-cognitive task & 10 & & & & 1.14 \\
\hline Sig. & & 0.061 & 0.068 & 0.37 & 0.07 \\
\hline
\end{tabular}




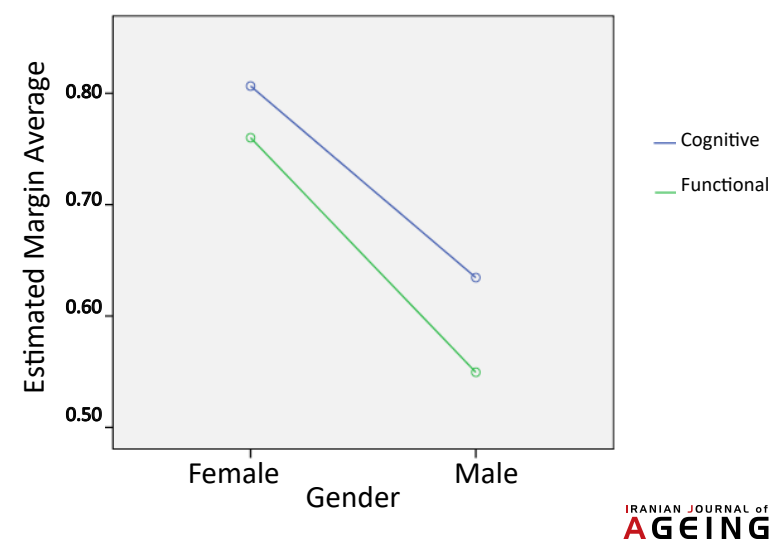

Figure 1. The mean position of COG under simultaneous cognitive and motor tasks

Post hoc test results presented in Table 1 showed that the mean position of COG in men with external attentional focus on motor and cognitive tasks, women with external attentional focus on motor task, and men with internal attentional focus on motor task (groups in first column) had a significant difference with other groups (columns 2-4). Also, the mean position of COG in groups placed in the first row was significantly better than that of groups in the second, third and fourth rows. There was a statistically significant difference between the mean performance of the groups placed in the second column with the groups in the first, third, and fourth columns.

Also, the mean performance of the groups in the third column was significantly different from that of groups placed in the first, second and fourth columns. Groups in the fourth column was also significantly different from other groups in terms of performance in learning instructions for postural control. In groups placed in the fourth column (women with external attentional focus on cognitive task, men with internal attentional focus on cognitive task, women with internal attentional focus on motor task, and women with internal attentional focus on cognitive task), the mean position of COG was significantly lower than that of the groups in first, second, and third columns.

Women with internal attentional focus on cognitive task had the poorest performance in learning postural control. Paired comparisons showed that elderly men and women with external attentional focus had a better postural control than those with internal focus. Men had a better attentional focus on cognitive and motor tasks than women (Figure 1).

\section{Conclusion}

Men who received instructions for external attentional focus had the best postural control, and both elderly men and women in performing motor tasks had better performance than those carrying out cognitive tasks. When focusing on motion tasks requiring physical accuracy, posture stability improves with respect to the compensatory mechanism in the central nervous system, but this effect is not observed in cognitive tasks [11]. Women received instructions for internal attentional focus on cognitive tasks had the weakest performance. The relations between the efficacy of postural control and concurrent cognitive demands is U-shaped [12].

Elderly men and women received instructions for external attentional focus had a better postural control than those received instructions for internal attentional focus. Based on constrained action hypothesis, when the attentional instruction directs attention to the movement effect in the environment, automatic control processes are facilitated [13]. Men in performing cognitive and motor tasks had better attentional focus than women. Decreased postural control in women may be due to their more muscular weakness compared to men [14].

\section{Ethical Considerations}

\section{Compliance with ethical guidelines}

The Ethics Committee of the Research Institute of Physical Education and Sport Sciences of Azad University of Tehran has confirmed this study.

\section{Funding}

This paper was extracted from the first author's thesis in the Department of Movement Behavior, Faculty of Physical Education and Sport Science, Central Tehran Branch, Islamic Azad University, Tehran,

\section{Conflict of interest}

The authors declared no conflict of interest.

Acknowledgements

We would like to thank the dear chairman of the Red Crescent Center and the staff of this center as well as the management of Yas Center and the elderly of this center. 


\title{
نقش جنسيت و كانون توجه بر كتترل وضعيت بدن سالمندان
}

\author{
•ماندانا سنكارى 'ـ، سيد محمدكاظم واعظ موسوى"، يروانه شمسىيور دهكردى"، مهدى نمازىزاده" \\ 1- كروه رفتار حركتى، دانشكده تربيت بدنى و علوم ورزشى، واحد تيران مركز، دانشكاه آزاد اسلامى، تهران، ايران.

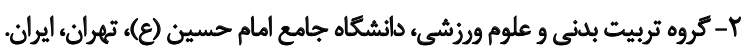

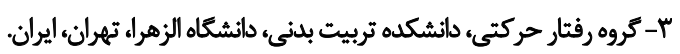

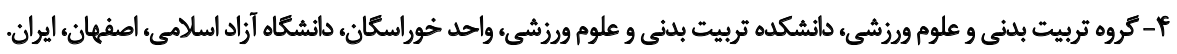

\section{באSי}

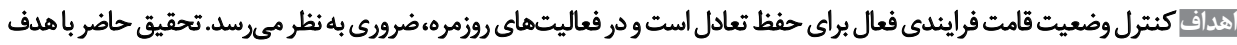

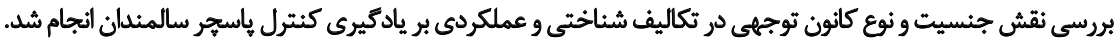

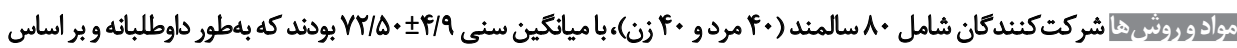

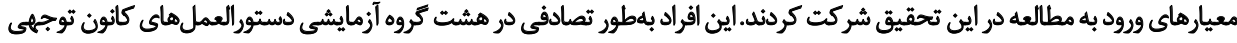

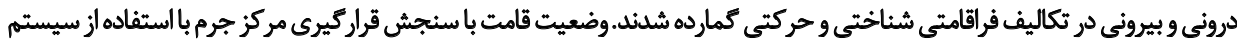

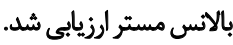

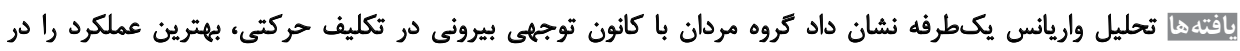

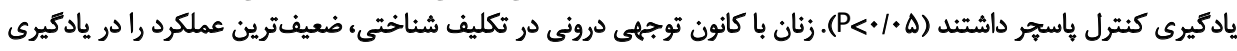

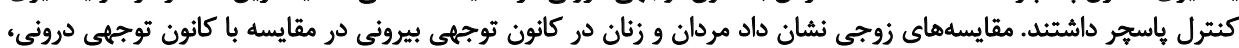

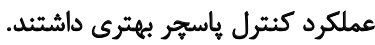

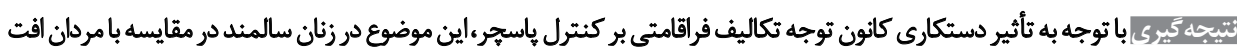

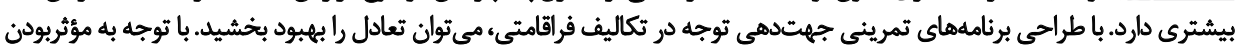

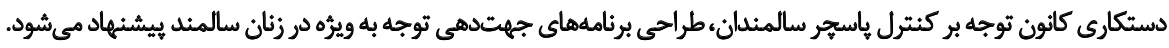

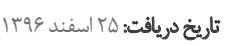

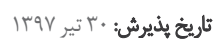

تئورى بردازش آكاهانه' نشان مى دهد توجه درونى به كنترل

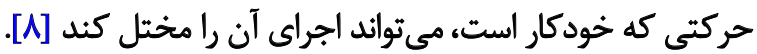

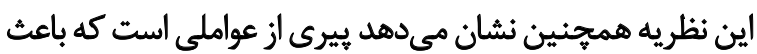

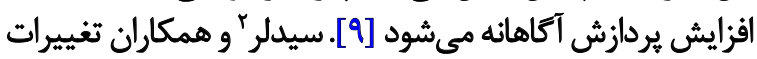

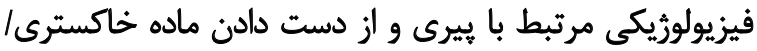

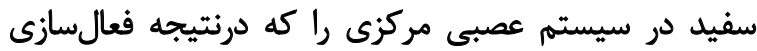

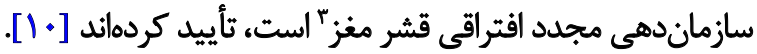

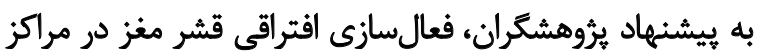

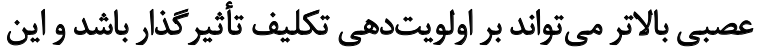

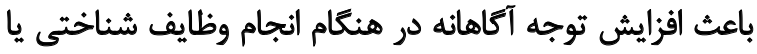

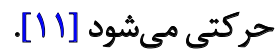

1. Theory of Reinvestment

2. Seidler

3. Differential-reorganized cortical activation

مقدمه

كنترل ياسجر مؤلفهاى جدايىنايذير از كنترل حركتى و فرايند

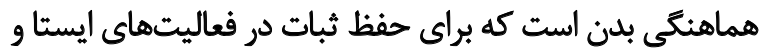

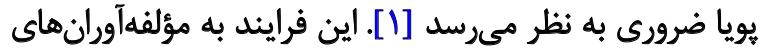

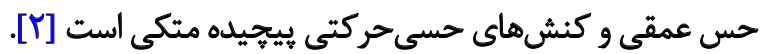

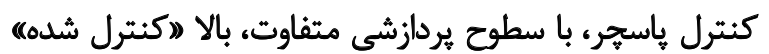

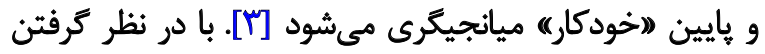

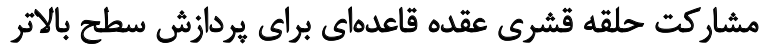

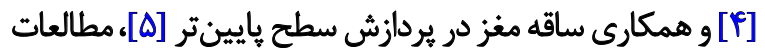

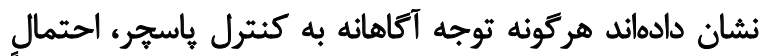

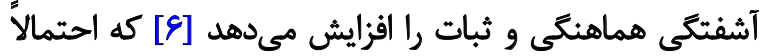

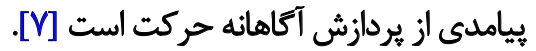

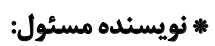

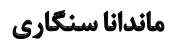

نشائى: تهران، دانشكاه آزاد اسلامى، واحد تهران مركز، دانشكده تربيت بدنى و علوم ورزشى، كروه رفتار حركتى.

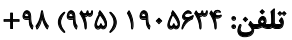
mandana.sangari@gmail.com يست الكترونيكي: 
مكىشود نظرات و نتايج همسانى وجود ندارد كه بتوان نظريهاي

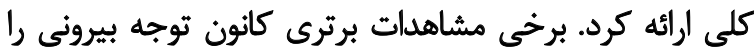

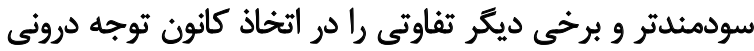

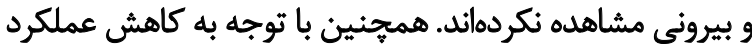

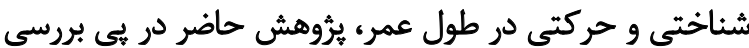

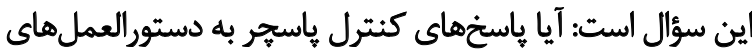
كانون توجهى تكاليف شناختى و حركتى، بين سالمندان زن و

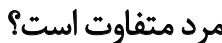

$$
\text { روش مطالعه }
$$

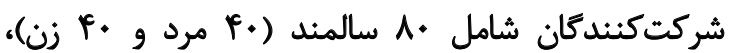

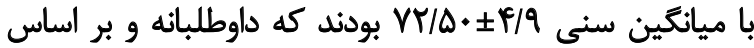

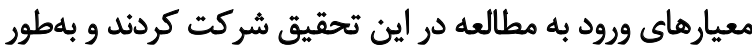

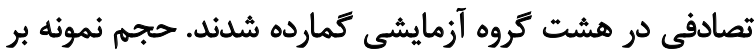

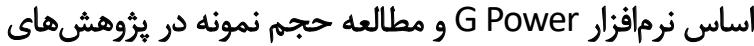

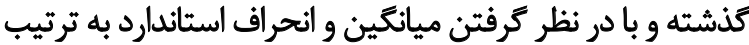

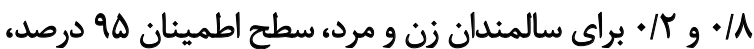

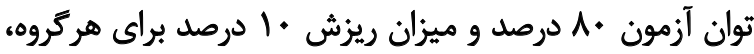

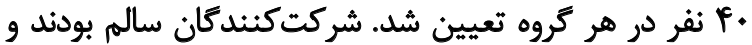

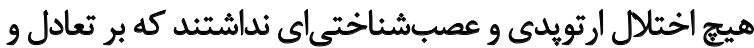

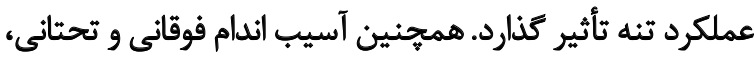

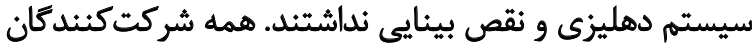

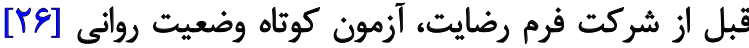

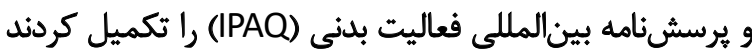

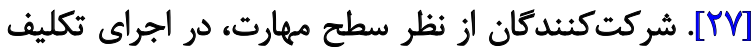

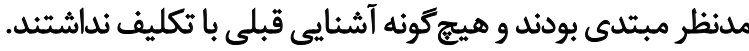
براى سنجش وضعيت قامت از سيستم بالانس مستر \استفاده

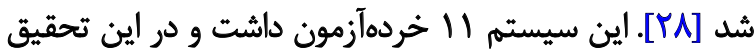

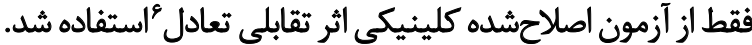

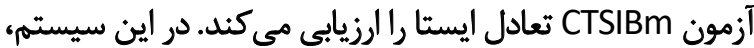

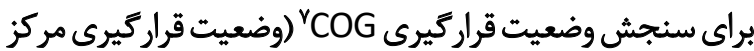

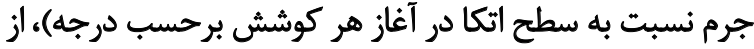

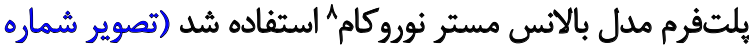

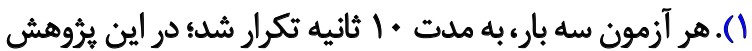

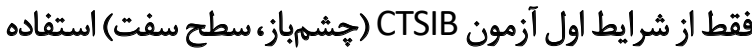
و ميانگين سه كوشش آزمون ارائه شد.

براي فراهم كردن شرايط تكليف حركتى، آزمودنىها استوانه

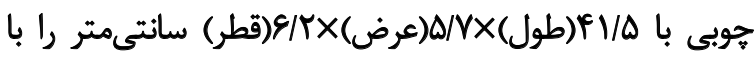

5. Master balance (SOT)

6. Modified Clinical Test of Sensory Interaction and Balance (mCTSIB)

7. COG Alignment

8. NeuroCom Balance Master
اكثرفعاليتهايروزانهنيازمندمديريت تكاليفحركتىشناختى

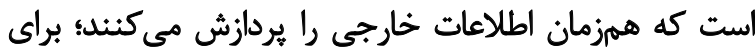

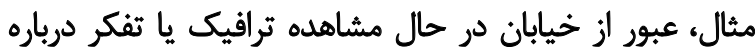

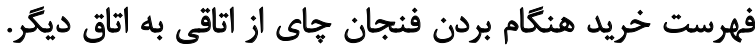

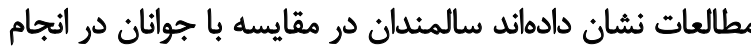

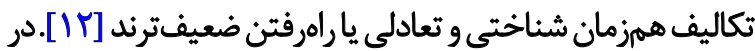

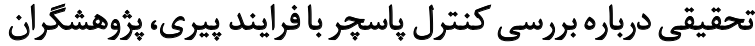

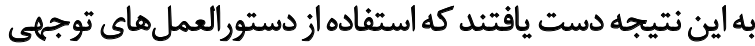

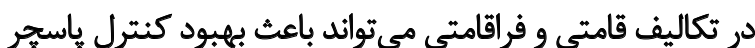

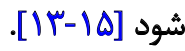

مطالعات اخير نشان داده است انجام تكاليف حركتى و

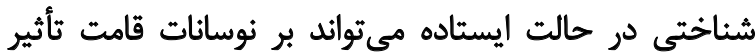

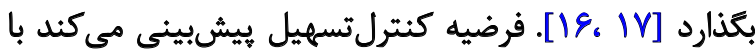

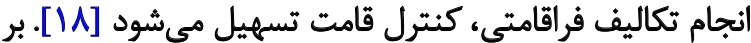

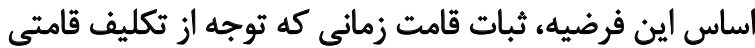

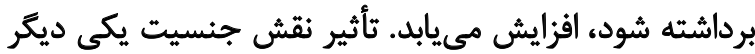

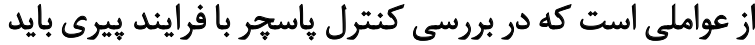

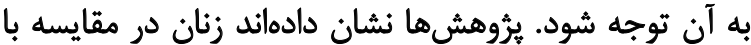

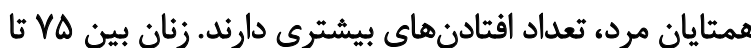

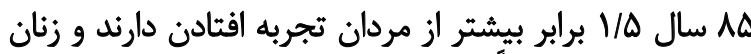

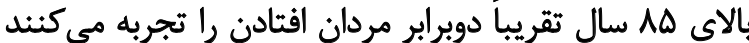

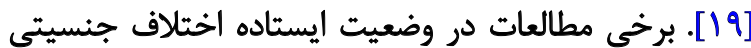

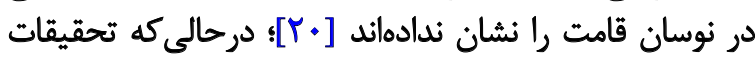

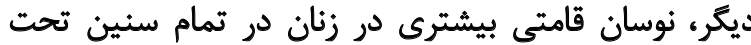

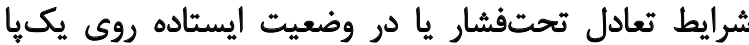

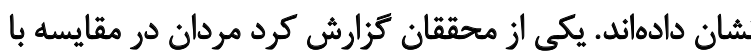

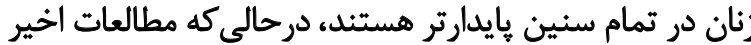

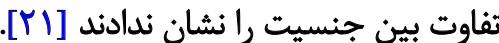
ولسون" و همكاران مشخص كردند تكاليف كنترل ياسجر

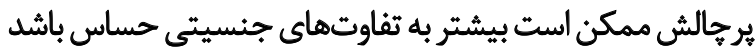

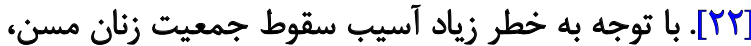

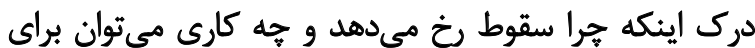

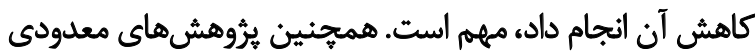

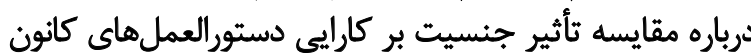
توجه تكاليف شناختى و حركتي انجام شده است. كانونى كردن

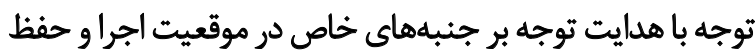

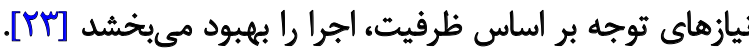

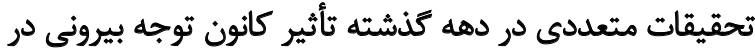

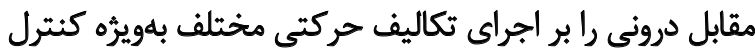

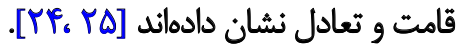
با مرور يروهشهاى موجود درباره اين موضوع، مشاهده 
ديكر تكميل شد. سيس شركت كنندكان سالمند كه سطح بايه

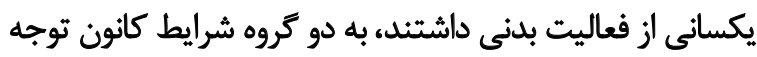

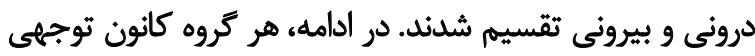

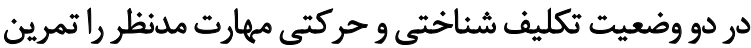

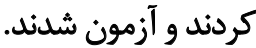

در كروه اول (سالمندان زن با دستورالعمل توجهي درونى دركي

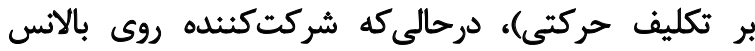

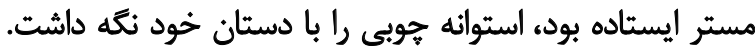

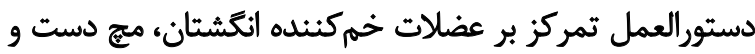

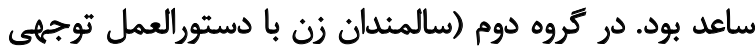
بيرونى بر تكليف حركتى)، به فرد دستور داده شد بر بر نتهيداشتن

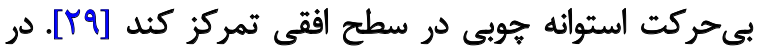

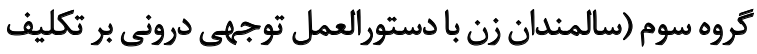

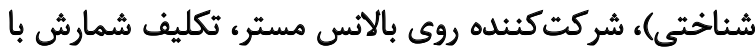
توالى سهتايى را انجام داد. دستور العمل تمركز

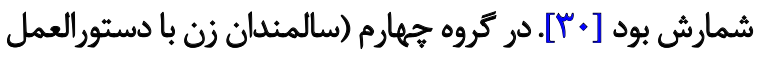

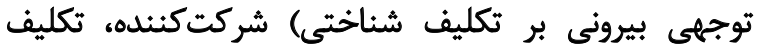

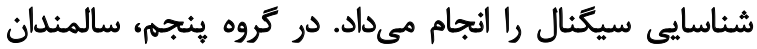

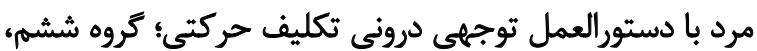

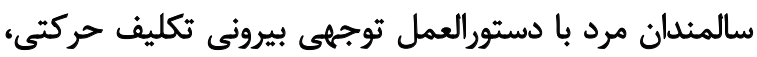

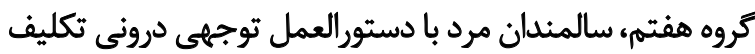

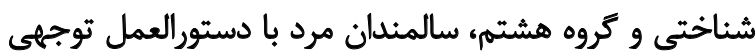

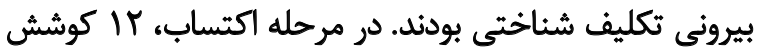

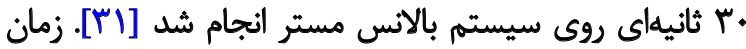

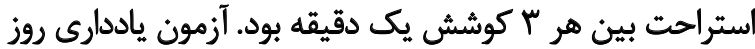

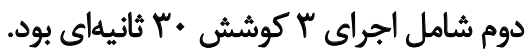
از آمار توصيفى براى شاخصهاي ميانكين، انحراف استاندارد

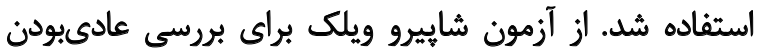

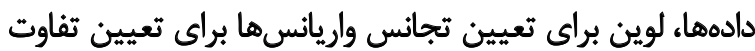

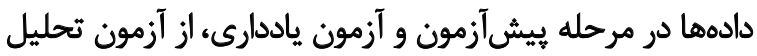

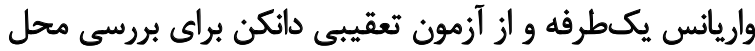

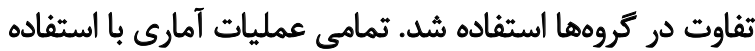

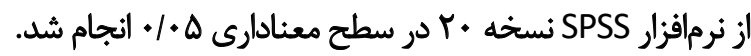

يافتّهنا

نتايج آزمون شاييروويلك نشان داد توزيع دادهها براي متغير

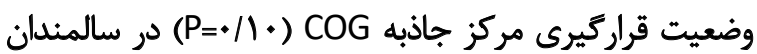

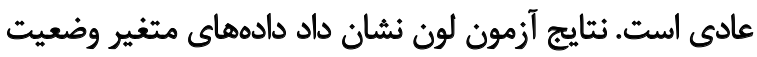

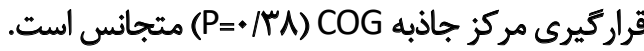

نتايج تحليل واريانس يكراهـ در مرحله بيشيش آزمون نشان

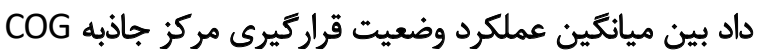

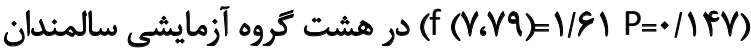

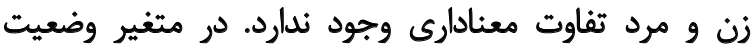

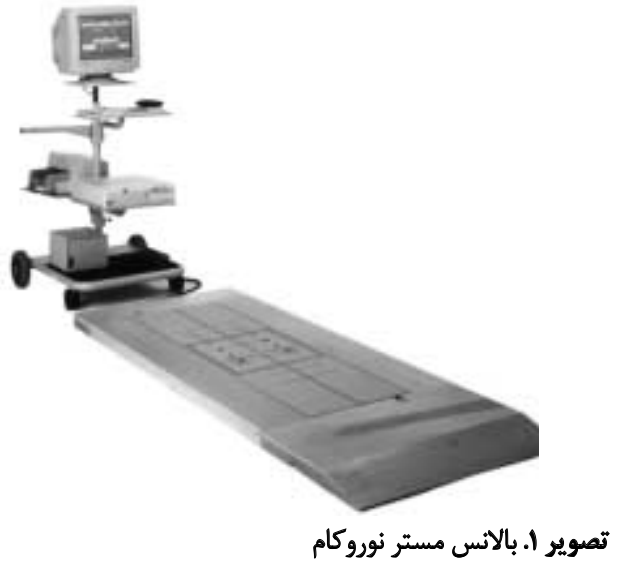

دستان خود در زاويه آرنج •9 درجه روبهروى شكمشان نكه

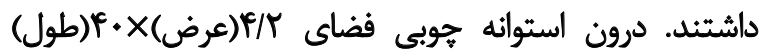

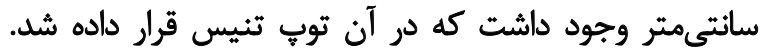

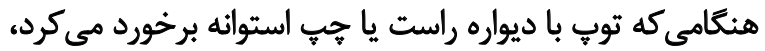

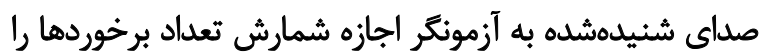

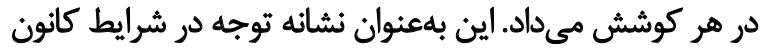

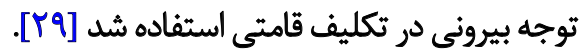
تكليف شناختى با دستورالعمل توجهى درونى شامل شمردن

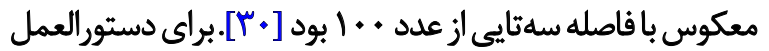

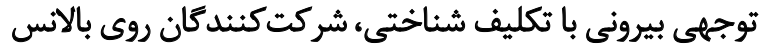

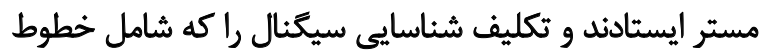

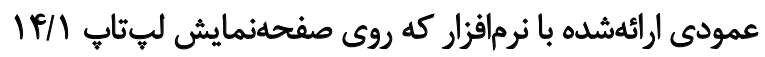

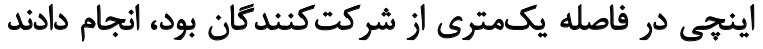

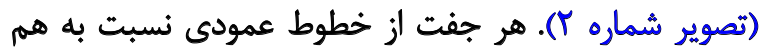

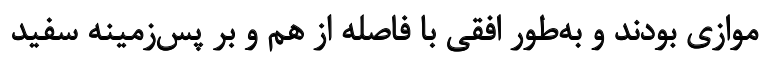
نمايش داده شدند [إب]. يكى روز قبل از جلسه تمرين،براى آزمودنى ها توضيحات كاملى

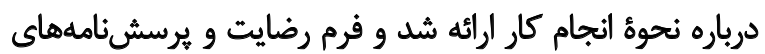

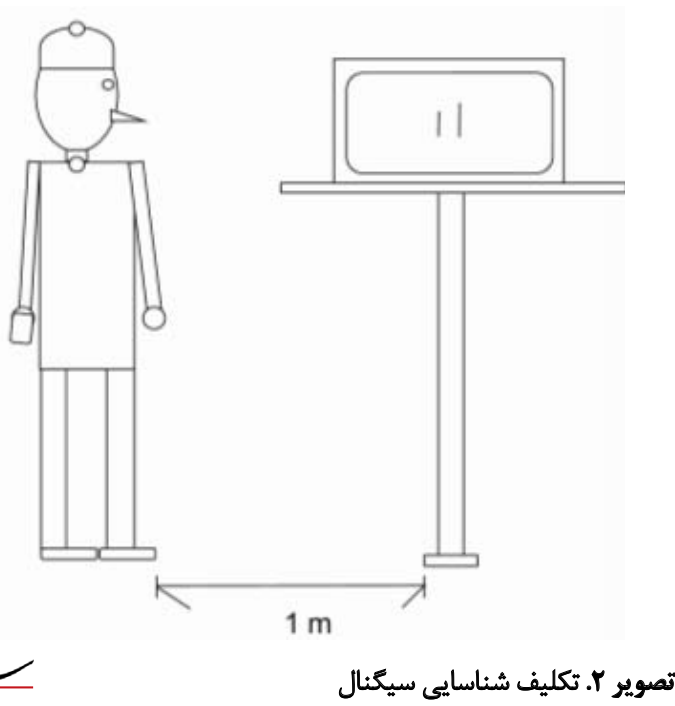


جدول ا. مقايسه وضعيت قراركيرى COG در هشت كروه آزمايشى سالمئدان

\begin{tabular}{|c|c|c|c|c|c|}
\hline $\mathbf{P}$ & $\mathbf{F}$ & ميانكين مجذورات & درجات آزادى & مجموع مجذورات & مثتغير \\
\hline & & $\cdot / A V$ & $\checkmark$ & $q / \cdot \gamma$ & \\
\hline \multirow[t]{2}{*}{$+1+.+1$} & r/৭ब &.$/ M T$ & $n$ & IQ/Ar & وضعيت قراركيرى COG \\
\hline & & & va & $r / 9 I$ & \\
\hline
\end{tabular}

닌

جدول r. نتايج أزمون تعقيبي دانكن براى مقايسه زوجى ميانكين وضعيت قراركيرى مركز جاذبه COG هشت كروه آزمايشى سالمندان در آزمون ياددارى

\begin{tabular}{|c|c|c|c|c|c|}
\hline \multicolumn{4}{|c|}{$\alpha=\star 1 \bullet \bullet$} & \multirow{2}{*}{$\mathbf{N}$} & \multirow{2}{*}{$\operatorname{lig}$} \\
\hline ستون جهارم & ستون سوم & ستون دوم & ستون اول & & \\
\hline & & &.$/ \pi$ & 1. & مرد- كانون توجهى بيرونى - تكليف عملكردى \\
\hline & &.$/ M F$ &.$/ M F$ & 1. & مرد- كانون توجهى بيرونى - تكليف شناختى \\
\hline & &.$/ M V$ &.$/ M V$ & 1. & نن - كانون توجهى ييرونى - تكليف عملكردى \\
\hline &.$/ 9 T^{2}$ &.$/ g e$ & $.19 T^{2}$ & 1. & مرد- كانون توجهى درونى - تكليف عملكردى \\
\hline.$/ N$ & $\cdot M$ & $\cdot M$ & $\cdot M$ & 1. & زن - كانون توجهى بيرونى-تكليف شثاختى \\
\hline.$/ \mathrm{NA}$ & - $/ \mathrm{NA}$ & - NA & & 1. & هرد- كائون توجهى درونى - تكليف شناختى \\
\hline.$/ A \Delta$ & / / $A \Delta$ & & & 1. & زن -كانون توجهى درونى - تكليف عملكردى \\
\hline Whe & & & & 1. & زن -كانون ثوجهى درونى - تكليف شناختى \\
\hline$\cdot 1 \cdot v$ & $\cdot / r V$ & .1 .81 & .1 .81 & & سطح معنادارى \\
\hline
\end{tabular}

تفاوتها از آزمون تعقيبى دايكن استفاده شد (جدول شماره r). نتايج آزمون تعقيبى نشان داد بين ميانكين وضعيت قراركيرى

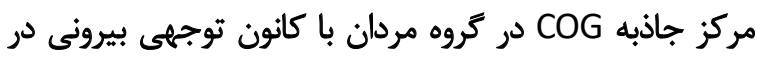

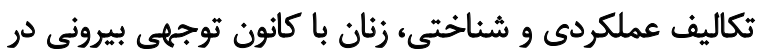

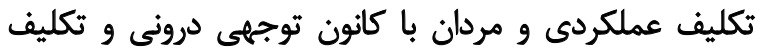

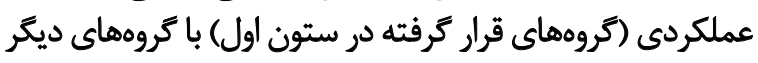

قرارتيرى مركز جاذبه COG براى بررسى محل تفاوتها در هشت

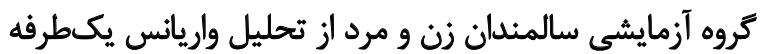
استفاده شد (جدول شماره (1) ). نتايج آزمون تحليل واريانس يكسطرفه نشان داد براى متغير مراني

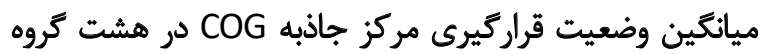

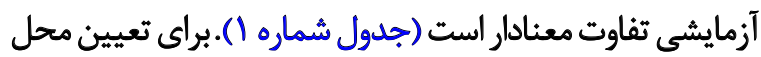

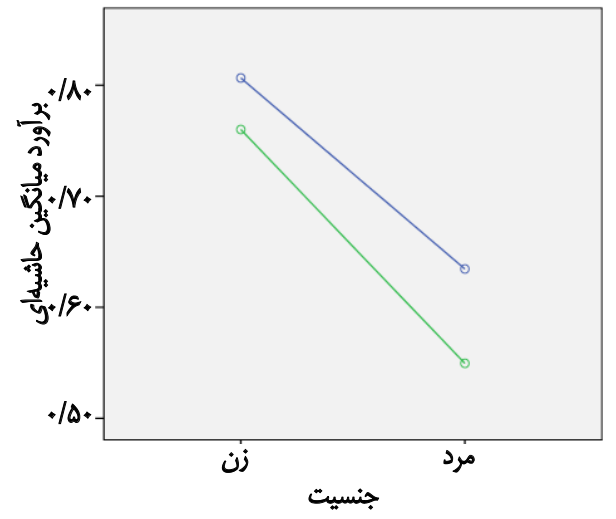

كالم

تصوير F. ميانكين قراركيرى مركز جاذبه COG با توجه به تكليف همزمان

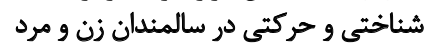

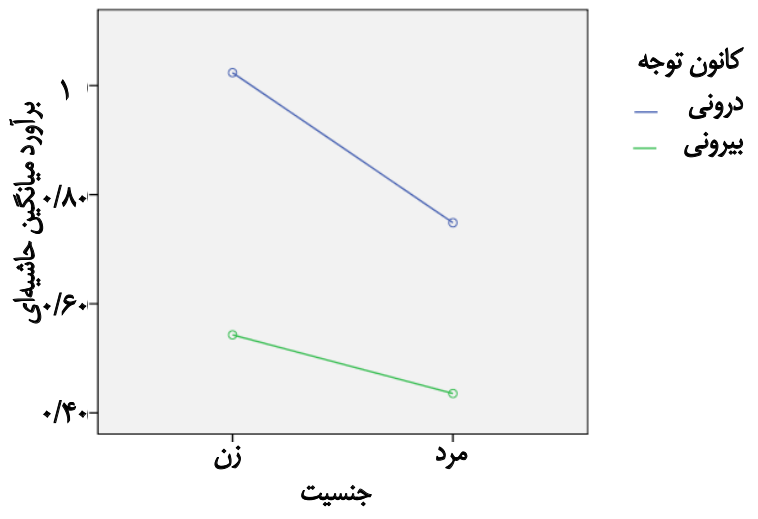

ill

تصوير r. ميائكين قراركيرى COG با توجه به كانون توجه در سالمندان 
باشد كه به صحت دقت فيزيكى نيازمند است، ثبات ياسجر باتوجه

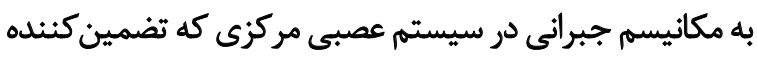

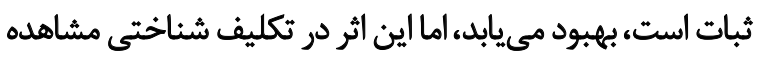

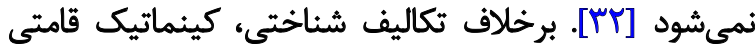

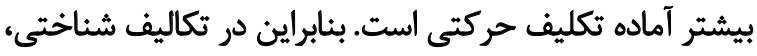

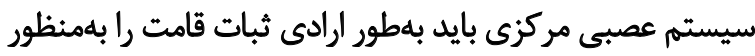

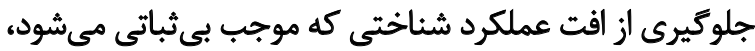

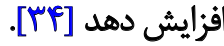

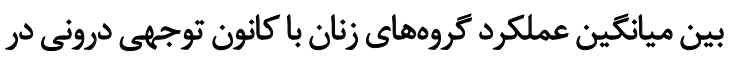

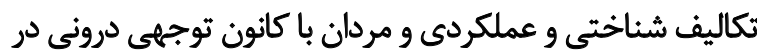

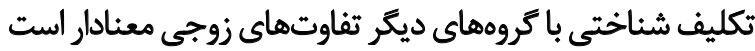

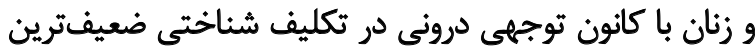

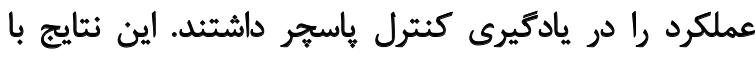

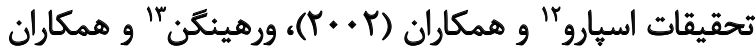

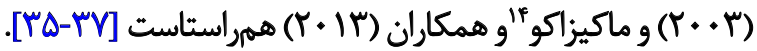

اوليورها در يروهش خود تأييد كرد تداخل بين فعاليت

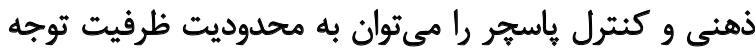

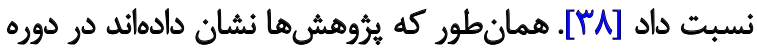

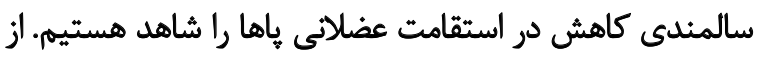

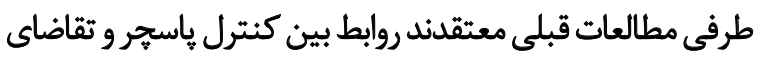

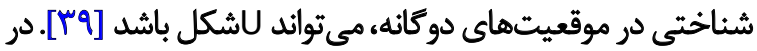

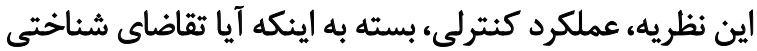

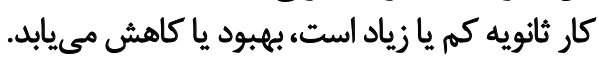

خو بس مى توان نتيجه كرفت افزايش بار شناختى در سالمندى كه

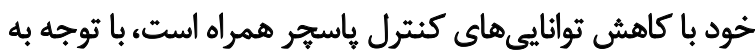

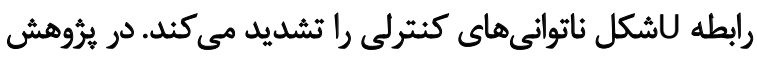

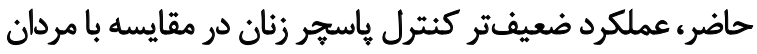

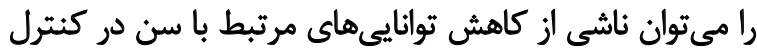

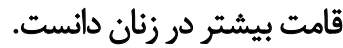

مردان و زنان در كانون توجهى بيروني، عملكرد كنترل ياسجر

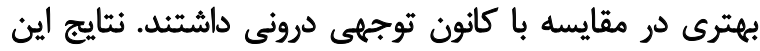

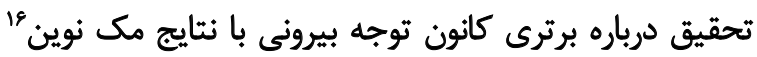

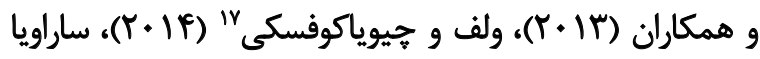

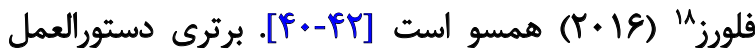
كانون توجهى بيرونى براي تكليف حركتى در آزمونئىها با بنتايج

\section{Sparrow}

13. Verhaeghen

14. Makizako

15. Olivier

16. McNevin

17. Wulf \& Chiviacowsky

18. Saraiva Flôres
(كروههاي قرار كرفته در ستونهاي دوم، سوم و جهارم)تفاوتهاي

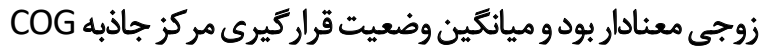

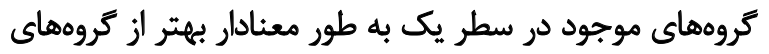

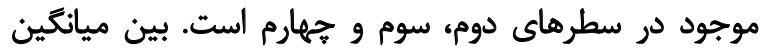

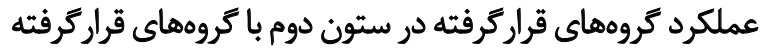

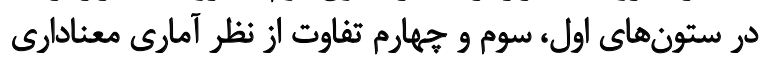

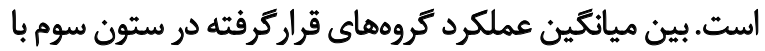

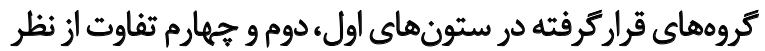

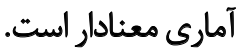

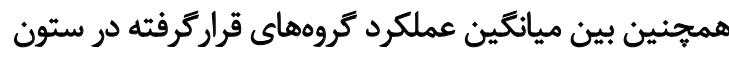

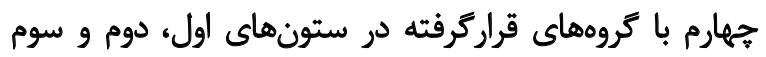

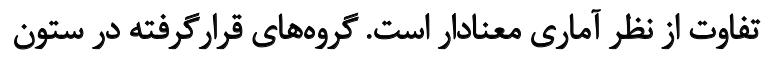

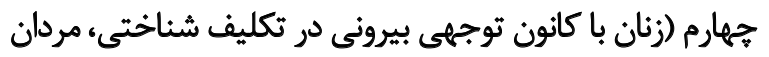

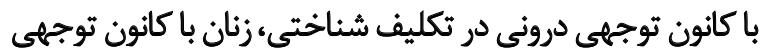

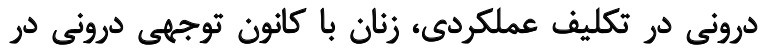

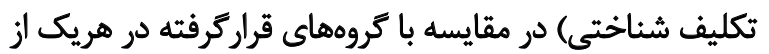

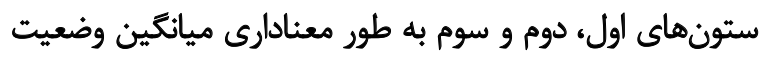

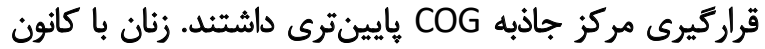

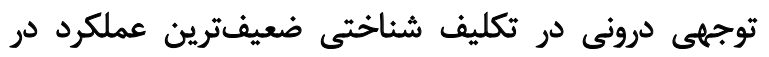

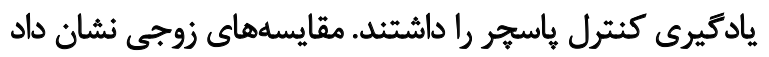

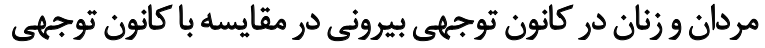

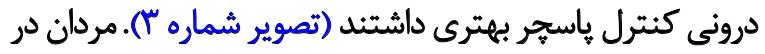

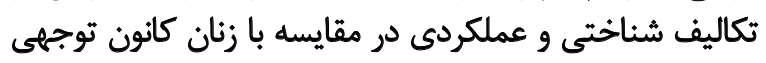
بهترى داشتند (تصوير شماره f). \&) هدف از انجام يُروهش حاضر بررسى نقش جنسيت و نو نوع كانون

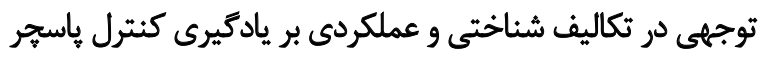

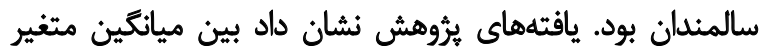

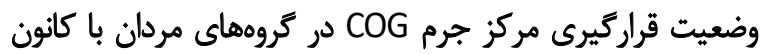

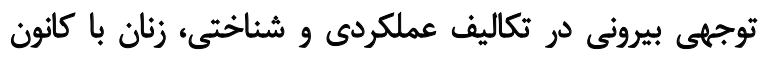

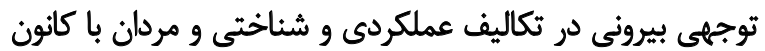

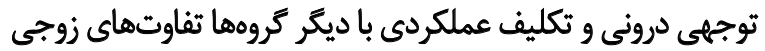

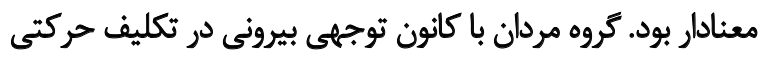

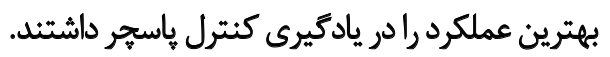

مقايسه ميانكَينها نشان داد هر دو كروه سالمندان در تكليف

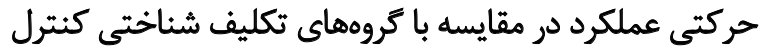

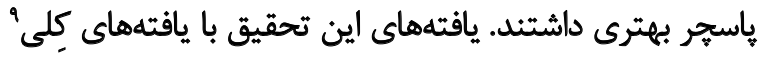

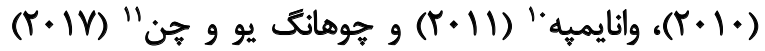

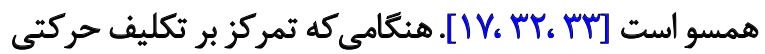

9. Kelly

10. Van Impe

11. Yu \& Huang 


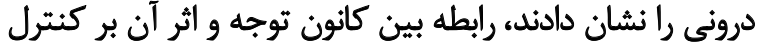

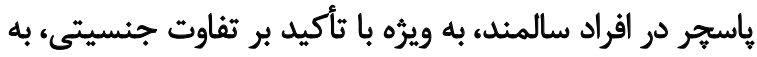

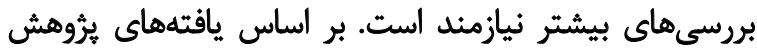

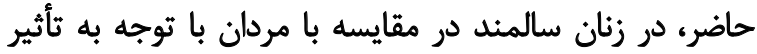

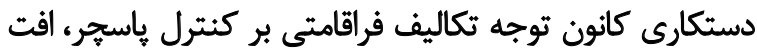

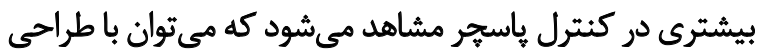

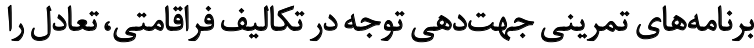

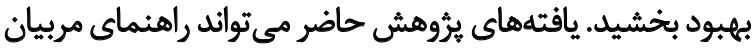

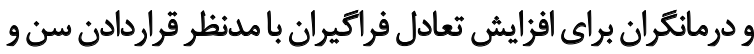

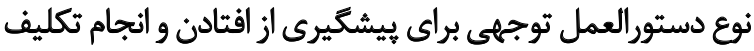

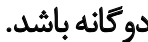

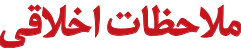

\section{ي ميروى از اصول اخلاق يثوهش}

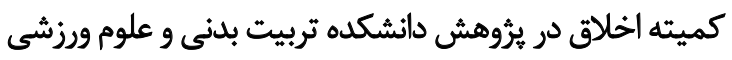

دانشعاه آزاد تهران مركز اين يثروهش را تأييد كرده است.

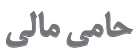

اين مقاله از رساله دكتراى خانم ماندانا سنكارى از كروه رفتار

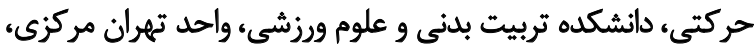

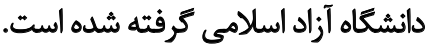

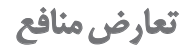

بنا بر اظهارنظر نويسندًان، تعرض منافعى وجود ندارد.

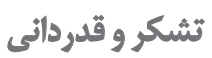

مطالعه حاضر در مركز كاردرمانى مركز هلالاحمر تهران و با بال

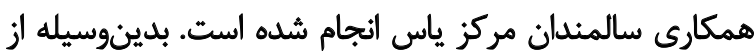

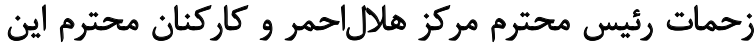

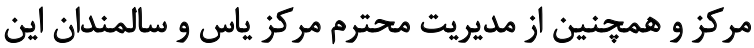

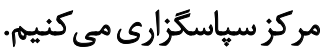

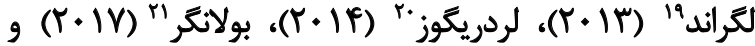

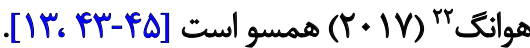

در تلاش براى توضيح علت برترى كانون توجه بيرونى در

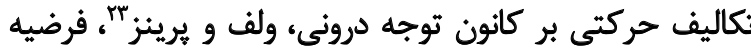

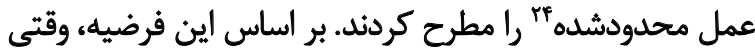

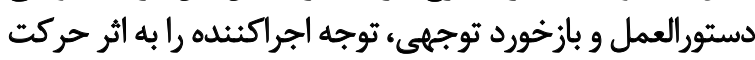

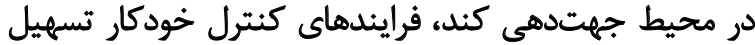

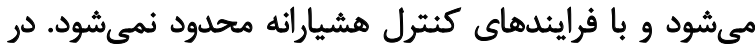

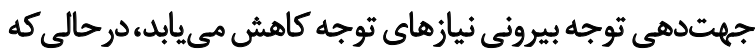

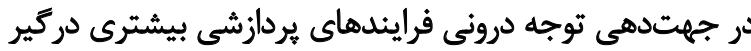

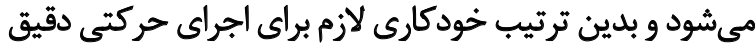

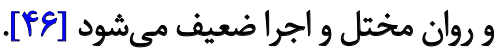

مردان سالمند در تكاليف شناختى و عملكردى در مقايسه با

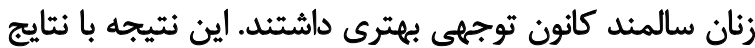

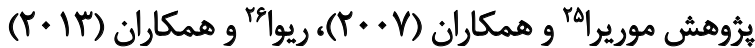

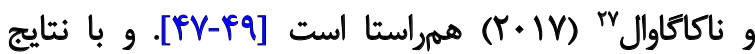

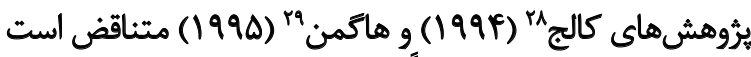

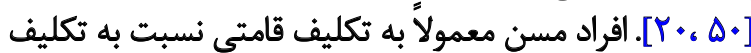

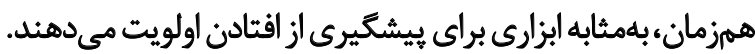

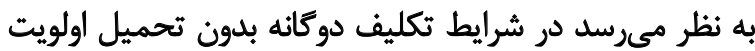

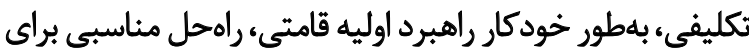

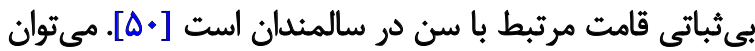

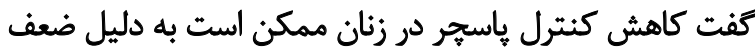

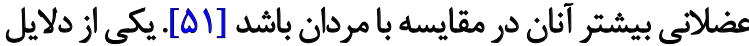

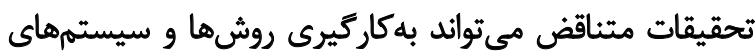
مختلف ارزيابى كنترل ياسجير باشد.

\section{نتيجهيكيرى نهايي}

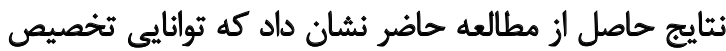

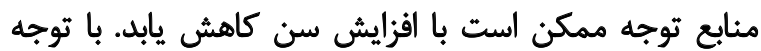

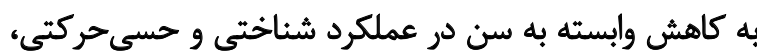

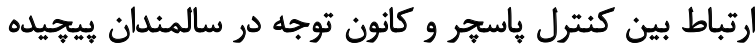

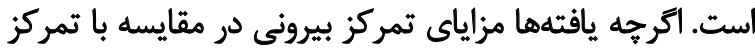

19. Legrand

20. Rodrigues

21. Boulanger

22. Huang

23. Wulf \& Prinz

24. Constrained Action Hypothesis

25. Moreira

26. Riva

27. Nakagawa

28. Colledge

29. Hageman 


\section{References}

[1] Wikstrom EA, Tillman MD, Smith AN, Borsa PA. A new forceplate technology measure of dynamic postural stability: The dynamic postural stability index. Journal of Athletic Training. 2005; 40(4):305-9. [PMID] [PMCID]

[2] Ghai S, Driller M, Ghai I. Effects of joint stabilizers on proprioception and stability: A systematic review and meta-analysis. Physical Therapy in Sport. 2017; 25:65-75. [DOI:10.1016/j. ptsp.2016.05.006]

[3] Boisgontier MP, Beets IA, Duysens J, Nieuwboer A, Krampe RT, Swinnen SP. Age-related differences in attentional cost associated with postural dual tasks: Increased recruitment of generic cognitive resources in older adults. Neuroscience \& Biobehavioral Reviews. 2013; 37(8):1824-37. [DOI:10.1016/j.neubiorev.2013.07.014]

[4] Jacobs J, Horak F. Cortical control of postural responses. Journal of Neural Transmission. 2007; 114(10):1339-48. [DOI:10.1007/ s00702-007-0657-0]

[5] Honeycutt CF, Gottschall JS, Nichols TR. Electromyographic responses from the hindlimb muscles of the decerebrate cat to horizontal support surface perturbations. Journal of Neurophysiology. 2009; 101(6):2751-61. [DOI:10.1152/jn.91040.2008]

[6] Masters R, Maxwell J. The theory of reinvestment. International Review of Sport and Exercise Psychology. 2008; 1(2):160-83. [DOI:10.1080/17509840802287218]

[7] Wulf G, McNevin N, Shea CH. The automaticity of complex motor skill learning as a function of attentional focus. The Quarterly Journal of Experimental Psychology: Section A. 2001; 54(4):114354. [DOI:10.1080/713756012]

[8] Masters RS. Knowledge, knerves and know-how: The role of explicit versus implicit knowledge in the breakdown of a complex motor skill under pressure. British Journal of Psychology. 1992; 83(3):343-58. [DOI:10.1111/j.2044-8295.1992.tb02446.x]

[9] Schaefer S, Schellenbach M, Lindenberger U, Woollacott M. Walking in high-risk settings: do older adults still prioritize gait when distracted by a cognitive task? Experimental Brain Research. 2015; 233(1):79-88. [DOI:10.1007/s00221-014-4093-8]

[10] Seidler RD, Bernard JA, Burutolu TB, Fling BW, Gordon MT, Gwin JT, et al. Motor control and aging: Links to age-related brain structural, functional, and biochemical effects. Neuroscience \& Biobehavioral Reviews. 2010; 34(5):721-33. [DOI:10.1016/j.neubiorev.2009.10.005]

[11] Talelli P, Ewas A, Waddingham W, Rothwell JC, Ward NS. Neural correlates of age-related changes in cortical neurophysiology. Neuroimage. 2008; 40(4):1772-81. [DOI:10.1016/j.neuroimage.2008.01.039]

[12] Wickens CD. Multiple resources and performance prediction. Theoretical Issues in Ergonomics Science. 2002; 3(2):159-77. [DOI:10.1080/14639220210123806]

[13] Huang CY, Chang GC, Tsai YY, Hwang S. An increase in postural load facilitates an anterior shift of processing resources to frontal executive function in a postural-suprapostural task. Frontiers in Human Neuroscience. 2016; 10. [DOI:10.3389/fnhum.2016.00420]

[14] Schaefer S. The ecological approach to cognitive-motor dualtasking: findings on the effects of expertise and age. Frontiers in Psychology. 2014; 5:1167. [DOI:10.3389/fpsyg.2014.01167]
[15] Yeh TT, Cinelli ME, Lyons J, Lee TD. Using a manipulation check to uncover age-related difference in focus of attention instruction during a balance task. Experimental Aging Research. 2016; 42(3):307-13. [DOI:10.1080/0361073X.2016.1156977]

[16] Wulf G. Attentional focus and motor learning: A review of 10 years of research. E-journal Bewegung und Training. 2007; 1(23):1-11.

[17] Yu SH, Huang CY. Improving posture-motor dual-task with a supraposture-focus strategy in young and elderly adults. PloS One. 2017; 12(2):e0170687. [DOI:10.1371/journal.pone.0170687]

[18] Stoffregen TA, Pagulayan RJ, Bardy BtG, Hettinger LJ. Modulating postural control to facilitate visual performance. Human Movement Science. 2000; 19(2):203-20. [DOI:10.1016/S01679457(00)00009-9]

[19] Stevens JA, Corso PS, Finkelstein EA, Miller TR. The costs of fatal and non-fatal falls among older adults. Injury Prevention. 2006; 12(5):290-5. [DOI:10.1136/ip.2005.011015]

[20] Colledge N, Cantley P, Peaston I, Brash H, Lewis S, Wilson J. Ageing and balance: the measurement of spontaneous sway by posturography. Gerontology. 1994; 40(5):273-8 [DOI:10.1159/000213596]

[21] Springer BA, Marin R, Cyhan T, Roberts H, Gill NW. Normative values for the unipedal stance test with eyes open and closed. Journal of geriatric physical therapy. 2007; 30(1):8-15 [DOI:10.1519/00139143-200704000-00003]

[22] Wolfson L, Whipple R, Derby CA, Amerman P, Nashner L. Gender differences in the balance of healthy elderly as demonstrated by dynamic posturography. Journal of Gerontology. 1994; 49(4):M160-7. [DOI:10.1093/geronj/49.4.m160]

[23] Magill RA, Anderson DI. Motor learning and control: Concepts and applications. New York: McGraw-Hill; 2007.

[24] Chiviacowsky S, Wulf G, Ávila L. An external focus of attention enhances motor learning in children with intellectual disabilities. Journal of Intellectual Disability Research. 2012; 57(7):627-34 [DOI:10.1111/j.1365-2788.2012.01569.x]

[25] Polskaia N, Richer N, Dionne E, Lajoie Y. Continuous cognitive task promotes greater postural stability than an internal or external focus of attention. Gait \& Posture. 2015; 41(2):454-8. [DOI:10.1016/j.gaitpost.2014.11.009]

[26] Folstein MF, Folstein SE, McHugh PR. "Mini-mental state": Apractical method for grading the cognitive state of patients for the clinician. Journal of Psychiatric Research. 1975; 12(3):189-98 [DOI:10.1016/0022-3956(75)90026-6]

[27] Craig CL, Marshall AL, Sjöström M, Bauman AE, Booth ML, Ainsworth $\mathrm{BE}$, et al. International physical activity questionnaire: 12-country reliability and validity. Medicine \& Science in Sports \& Exercise. 2003; 35(8):1381-95. [DOI:10.1249/01. MSS.0000078924.61453.FB]

[28] Namba T, Tabuchi A. Study on vision and static physical balance function Report 1: Healthy young and middle-elder people. Kawasaki Journal of Medical Welfare. 2015; 20(2):42-53.

[29] Wulf G, Weigelt M, Poulter D, McNevin N. Attentional focus on suprapostural tasks affects balance learning. The Quarterly Journal of Experimental Psychology: Section A. 2003; 56(7):1191211. [DOI:10.1080/02724980343000062] 
[30] Burcal CJ, Drabik EC, Wikstrom EA. The effect of instructions on postural-suprapostural interactions in three working memory tasks. Gait \& Posture. 2014; 40(2):310-4. [DOI:10.1016/j.gaitpost.2014.04.203]

[31] Chiviacowsky S, Wulf G, Wally R. An external focus of attention enhances balance learning in older adults. Gait \& Posture. 2010; 32(4):572-5. [DOI:10.1016/j.gaitpost.2010.08.004]

[32] Kelly VE, Janke AA, Shumway-Cook A. Effects of instructed focus and task difficulty on concurrent walking and cognitive task performance in healthy young adults. Experimental Brain Research. 2010; 207(1-2):65-73. [DOI:10.1007/s00221-010-2429-6]

[33] Van Impe A, Coxon J, Goble D, Wenderoth N, Swinnen S. Agerelated changes in brain activation underlying single-and dualtask performance: Visuomanual drawing and mental arithmetic. Neuropsychologia. 2011; 49(9):2400-9. [DOI:10.1016/j.neuropsychologia.2011.04.016]

[34] Marone JR, Patel PB, Hurt CP, Grabiner MD. Frontal plane margin of stability is increased during texting while walking. Gait \& Posture. 2014; 40(1):243-6. [DOI:10.1016/j.gaitpost.2014.04.188]

[35] Makizako H, Furuna T, Ihira H, Shimada H. Age-related differences in the influence of cognitive task performance on postural control under unstable balance conditions. International Journal of Gerontology. 2013; 7(4):199-204. [DOI:10.1016/j. ijge.2013.01.014]

[36] Sparrow W, Bradshaw EJ, Lamoureux E, Tirosh O. Ageing effects on the attention demands of walking. Human Movement Science. 2002; 21(5-6):961-72. [DOI:10.1016/S0167-9457(02)001549]

[37] Verhaeghen P, Steitz DW, Sliwinski MJ, Cerella J. Aging and dual-task performance: a meta-analysis. Psychology and Aging. 2003; 18(3):443-60. [DOI:10.1037/0882-7974.18.3.443]

[38] Olivier I, Cuisinier R, Vaugoyeau M, Nougier V, Assaiante C. Age-related differences in cognitive and postural dual-task performance. Gait \& Posture. 2010; 32(4):494-9. [DOI:10.1016/j.gaitpost.2010.07.008]

[39] Vuillerme N, Nougier V, Camicioli R. Veering in human locomotion: Modulatory effect of attention. Neuroscience letters. 2002; 331(3):175-8. [DOI:10.1016/S0304-3940(02)00876-5]

[40] Flôres FS, Menezes KM, Katzer JI. [Influence of gender on attention and learning of motor skills (Portuguese)]. Journal of Physical Education. 2016; 27(1):2706. [DOI:10.4025/jphyseduc.v27i1.2706]

[41] McNevin N, Weir P, Quinn T. Effects of attentional focus and age on suprapostural task performance and postural control. Research Quarterly for Exercise and Sport. 2013; 84(1):96-103. [DOI:1 0.1080/02701367.2013.762321]

[42] Wulf G, Chiviacowsky S, Drews R. External focus and autonomy support: Two important factors in motor learning have additive benefits. Human Movement Science. 2015; 40:176-84. [DOI:10.1016/j.humov.2014.11.015]

[43] Boulanger M, Giraudet G, Faubert J. Interaction between the oculomotor and postural systems during a dual-task: Compensatory reductions in head sway following visually-induced postural perturbations promote the production of accurate double-step saccades in standing human adults. PLoS One. 2017; 12(3):e0173678. [DOI:10.1371/journal.pone.0173678]

[44] Legrand A, Mazars KD, Lazzareschi J, Lemoine C, Olivier I, Barra J, et al. Differing effects of prosaccades and antisaccades on postural stability. Experimental Brain Research. 2013; 227(3):397405. [DOI:10.1007/s00221-013-3519-z]

[45] Rodrigues ST, Polastri PF, Carvalho JC, Barela JA, Moraes R, Barbieri FA. Saccadic and smooth pursuit eye movements attenuate postural sway similarly. Neuroscience Letters. 2015; 584:292-5. [DOI:10.1016/j.neulet.2014.10.045]

[46] Wulf G, Prinz W. Directing attention to movement effects enhances learning: A review. Psychonomic Bulletin \& Review. 2001; 8(4):648-60. [DOI:10.3758/BF03196201]

[47] Riva D, Mamo C, Fanì M, Saccavino P, Rocca F, Momenté M, et al. Single stance stability and proprioceptive control in older adults living at home: Gender and age differences. Journal of Aging Research. 2013; 2013:1-14. [DOI:10.1155/2013/561695]

[48] Moreira MD, Costa AR, Caldas CP. The association between nursing diagnoses and the occurrence of falls observed among eldery individuals assisted in an outpatient facility. Revista Latino-Americana de Enfermagem. 2007; 15(2):311-7. [DOI:10.1590/ S0104-11692007000200018]

[49] Nakagawa HB, Ferraresi JR, Prata MG, Scheicher ME. Postural balance and functional independence of elderly people according to gender and age: cross-sectional study. Sao Paulo Medical Journal. 2017; 135(3):260-5. [DOI:10.1590/1516-3180.2016.0325280217]

[50] Hageman PA, Leibowitz JM, Blanke D. Age and gender effects on postural control measures. Archives of Physical Medicine and Rehabilitation. 1995; 76(10):961-5. [DOI:10.1016/S00039993(95)80075-1]

[51] Berger L, Bernard-Demanze L. Age-related effects of a memorizing spatial task in the adults and elderly postural control. Gait \& Posture. 2011; 33(2):300-2. [DOI:10.1016/j.gaitpost.2010.10.082] 
